CHARACTERIZATION OF SPHERICAL AND PSEUDO-SPHERICAL SETS OF POINTS

\author{
A Thesis \\ Presented to the Faculty of \\ The Rice Institute in partial fulfillment of \\ the requirements for \\ the degree of Master of Arts \\ by \\ George Alvin Garrett
}


CHARACTERIZATION OF SPHERICAL AND PSEUDO-SPHERICAL SETS OF POINTS

A semi-metric space is defined to be a set of undefined elements, called "points", ouch that for any two elements p, q of the set there corresponds a not negative real number $p \dot{q}$, called the "distance" between the points $p$ and $q$, which satisfies the following postulates:

(1). $p q=q p$;

(2). $p q=0$ if and only if the points $p$ and $q$ are identical. Two pairs of points $p, q ; p\} q^{\prime}$ such that $p q=p^{\prime} q^{\prime}$ are said to be congruent. A mapping of a set $S$ upon a set $S^{\prime}$ is called a congruent mapping if to each pair of points of $S$ there corresponds a congruent pair of points of S'. Two sets $S$ and $S^{\prime}$ are said to be congruent provided there exists a congruent mapping of one upon the other. The nature of the investigations presented here justifies their division into two sections, a description of which follows.

Section I. The Circle. A set is said to be d-cyclic if it is congruent to a subset of a circle of metric diameter $d$, the distance between any two points of the circle being defined to be the length of the shorter arc joining them; while a set is pseudo d-cyclic if each three points of the set is d-cyclic, but the set itself is not d-cyclic. D-cyclic and pseado d-cyclic sets of points are characterized by means of distance relations expressible in determinantal form., The principal theorem concerming pseudo d-cyclic sets shows that such sets are equilateral provided they contain more than four points, and no four of tho points form a convex tripod*. This theorem is equivalent to a new

* Four points are said to form a convex tripod if one of the points lies between each of the three pairs of points contained in the remaining three points. The point $q$ is said to lie between the points $p$ and $r$ if $p q+q r=p r$. 
theorem on nth-order determinants of a certain class. Using synthetic methods; I. M. Blumenthal has proved most of the theorems contained in this section*. Finally, a method of construction of the three kinds of .pseudo d-cyclio quadruples is obtained.

Section II. The Sphere $S_{2, t}$. The two-dimensional spherical

apace $S_{2, r}$ consists of the points of the surface of a three-dimensional sphere of radius $r$ in a Euclidean epace of three dimensiona, with the distance between two points of the surface defined to be the length of the shorter arc of a great circle through the two points. The $S_{2, k}$ is characterized among general semi-metric spaces by means of four principal theorems. A set of points which is congruent to a subset of the $S_{2, r}$ is said to be r-apheric. A sot such that each four points of the set is r-spheric while the set itself is not r-spheric is said to be pseudo r-spheric. A method of constructing pseudo r-spheric quintuples is obtained and some of their properties are derived. A theorem concerning pseudo r-spheric sets similar to the "equilateral" theorem proved for pseudo d-cyclic sets has not yet been proved.

\section{The Circle}

Let $p_{i}$ and $p_{j}$ be any two points of a semi-metric space. Wo denote by $\alpha_{i j}$ the angle $\frac{p_{i} p_{j}}{r}$ radians, where $r$ is the radius (Euclidean) of the circle of metric diameter $d ; 1 . \theta_{0} d=\pi r$.

* These theorems are contained in two papers published in the American Journal of Mathematica; "A Complete Characterization of Proper Psoudo d-Cyclic sets of Points", Vol. 54 (1932), pp. 387-396; and

"Concernimg Regular Pseudo d-Cyclic Sets", Vol. 54, pp. 729-738. 
If $p_{1}, p_{2}, \ldots, p_{n}$ are $n$.points of a semi-metric space, we denote .. by $\Delta\left(p_{1}, p_{2}, \ldots, p_{n}\right)$ the axisymmetric determinant

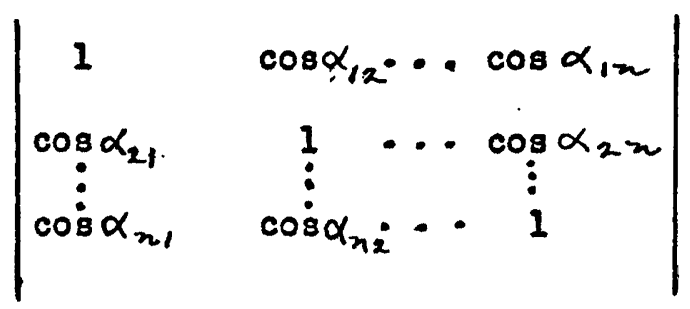

Theorem 1. Three points $\mathrm{p}_{1}, \mathrm{p}_{2}, \mathrm{p}_{3}$ of a semi-metric space are d-cyclic if and only if $0<\alpha_{i j} \leqslant \mathbb{T}$ and $\Delta\left(p_{1}, p_{z}, p_{3}\right)=0 .(i, j=1,2,3)$.

Evaluating the determinant $\triangle\left(p_{1}, p_{2}, p_{3}\right)$, we have

$$
\begin{aligned}
& \Delta\left(p_{1}, p_{2}, p_{1}\right)=1-\cos ^{2} \alpha_{12}-\cos ^{2} \alpha_{13}-\cos ^{2} \alpha_{23}+2 \cos \alpha_{12} \cos \alpha_{13} \cos \alpha_{23} \\
& =-\left[\cos \alpha_{23}-\cos \left(\alpha_{12}+\alpha_{13}\right)\right]\left[\cos \alpha_{i_{3}}-\cos \left(\alpha_{12}-\alpha_{13}\right)\right] \\
& =4 \sin \frac{\alpha_{12}+\alpha_{25}+\alpha_{13}}{2} \sin \frac{\alpha_{12}+\alpha_{12}-\alpha_{12}}{2} \sin \frac{\alpha_{12}-\alpha_{20}+\alpha_{13}}{2} \sin \frac{-\alpha_{12}+\alpha_{23}+\alpha_{13}}{2}
\end{aligned}
$$

We shall prove first that if $0<\alpha_{i j} \leqslant \pi$ and $\Delta\left(p, p_{2}, p_{3}\right)=0$, the points $p_{1}, p_{2}, p_{3}$ are d-cyclic. If $\Delta\left(p,, p_{2}, p_{3}\right)=0$, then by $(1)$ one of the following conditions mast subsist:

$$
\begin{aligned}
& {\left[\alpha_{12}+\alpha_{23}+\alpha_{13}=2 k \pi\right] ;\left[\alpha_{12}+\alpha_{23}-\alpha_{13}=2 k \pi\right] ;} \\
& {\left[\alpha_{12}-\alpha_{23}+\alpha_{13}=2 k \pi\right] ;\left[\alpha_{12}+\alpha_{23}+\alpha_{13}=2 k \pi\right] .}
\end{aligned}
$$

where $k$ is an integer. Since $0<\alpha_{i j} \leqslant \pi$, the only possible value for $k$ in the first of these equations is 1 , while in the three remaining equations the only possible value for $k$ is 0 . Hence, the four equations are equivalent to the following:

$$
p_{1} p_{2}+p_{2} p_{3}+p_{1} p_{3}=2 d ; p_{1} p_{2}+p_{2} p_{3}=p_{1} p_{3} ; p_{1} p_{2}+p_{1} p_{3}=p_{2} p_{3} ; p_{1} p_{3}+p_{2} p_{3}=p_{1} p_{2} \text {. }
$$

But, when one of these conditions exists, the points $p, p_{2}, p_{3}$ are d-cyclic*; and the sufficiency of the theorem is proved.

* L. H. Blumenthal, "A Complete Characterization of Proper Psoudo d-Cyclic Sets of Points", American Journal of Mathematics, Vol. 54, p. 388 
If $p_{1}, p_{2}, p_{3}$ are $d-c y c l i c$, then $0<p_{i} p_{j} \leqslant d$ and one of the conditions (2) subsists*. Hence, one of the factors in (1) vanishes. Thus, we have $0<\alpha_{i j} \leq \mathbb{T}$ and $\Delta\left(p_{1}, p_{2}, p_{3}\right)=0$, which establishes the necessity of the theorem.

From (1) we have immediately the following corollary: Corollary. The determinant $\Delta\left(p_{1}, p_{2}, p_{3}\right)$ is zero if and only if one of the relations

$$
\alpha_{12}+\alpha_{23}+\alpha_{13}=2 \pi ; \alpha_{12}+\alpha_{23}=\alpha_{13} ; \alpha_{12}+\alpha_{13}=\alpha_{23} ; \alpha_{23}+\alpha_{13}=\alpha_{12} ;
$$

is satiafied.

Definition. Three points of a semi-metric space are said to be circular provided the points are congruent to three points of some circlo.

Theorem2. A necessary and sufficient condition that three points p, p, p, of a semi-metric space be circular is that the points satisfy the triangular inequality.

For if $p_{1}, p_{2}, p_{3}$ are congruent to three points of a circle, then the points either are linearth in which case the triangular inequality is obviously satiafied) or else they satisfy the relation

$$
p_{1} p_{2}+p_{2} p_{3}+p_{1} p_{3}=2 d
$$

where $d$ is the metric diameter of the circle. Suppose $p_{1}, p_{2}, p_{3}$ do not satisfy the triangular inequality. We may assume the labeling so that $p_{1} \dot{p}_{2}>p_{2} p_{3}+p_{1} p_{3}$

Adding (1) and (2) we have $p, p_{2}>d$, which is impossible, since $d$ is the metric diameter of the circle containing the three points congruent to $p_{1}, p_{2}, p_{3}$. Hence, the necessity of the condition is established. Suppose now that $p_{1}, p_{2}, p_{3}$ satisfy the triangular inequality. two.

* Loc. cit.

f Three points are said to be linear if one is between the other 
If the triple is linear, then $p_{1}, p_{2}, p_{3}$ are congruent to three points of a circle of metric diameter $d$, where $d$ is greater than or equal to the largest of the numbers $p, p_{2}, p_{2} p_{3}, p, p_{3}$. If the triple is not linear, we may write

$$
p_{1} p_{2}+p_{2} p_{3}+p_{1} p_{3}=2 d^{\prime}
$$

Then the points are congruent to three points os a circle of metric diameter $d^{\prime}$. To show this we need only note that each of the distances $p_{1} p_{2}, p_{2} p_{3}, p_{1} p_{3}$, is less than $d^{\prime}$. For suppose pne of these distances, say $p_{1} p_{z}$, is hot less than $d^{\prime}$. Then

$$
\begin{aligned}
& p_{1} p_{2}+p_{2} p_{3}+p_{1} p_{3} \leqslant 2 p_{1} p_{2} ; \text { that is } \\
& p_{2} p_{3}+p_{1} p_{3} \leq p_{1} p_{2}
\end{aligned}
$$

But this is impossible since the points $p_{1}, p_{2}, p_{3}$ are not linear and the triangular inequality holds.

Using this result one may define a metric space as a semimetric space each three points of which is circular.

Theorem 2. Four points $p_{1}, p_{2}, p_{3}, p_{4}$ of a semi-metric space are d-cyclic if and only if each three of the points is d-cyclic and $\Delta\left(p_{1} p_{2} p_{2} p_{4}\right)=0$

To prove the necessity of the conditions we suppose that $p_{1}, p_{2}$, $p_{3}, p_{4}$ are congruent to four points $p_{1}^{\prime}, p_{2}^{\prime}, p_{3}^{\prime}, p_{4}^{\prime}$ of the circle. Then each three of the points is congruent to three points of the circle, and we have only to show that $\Delta\left(p_{1}, p_{2}, p_{3}, p_{4}\right)=0$. We take as the origin of a two-dimensional rectangular coordinate system the center of the circle through $p_{1}^{\prime}, p_{2}^{\prime}, p_{3}^{\prime}, p_{4}^{\prime}$ - Denoting by $A_{i}$ the cosine of the angle between the $x$-axis and the line joining the origin with $p_{i}^{!}$, and by $B_{i}$ the cosine of the angle between the $y$-axis and this line, we. may write 


$$
\begin{aligned}
\cdot \cos \alpha_{i j} & =A_{i} A_{j}+B_{i} B_{j} ; \\
1 & =A_{i}^{2}+B_{i}^{2} .
\end{aligned}
$$

Employing these two relations we may express $\Delta\left(p_{1}, p_{2}, p_{3}, p_{4}\right)$. in the form

$$
\begin{aligned}
\Delta\left(p_{1}, p_{2}, p_{3}, p_{4}\right) & =\left|\begin{array}{cccc}
A_{1}^{2}+B_{1}^{2} & A_{1} A_{2}+B_{1} B_{2} & A_{1} A_{3}+B_{1} B_{3} & A_{1} A_{4}+B_{1} B_{4} \\
A_{2} A_{1}+B_{2} B_{1} & A_{2}^{2}+B_{2}^{2} & A_{2} A_{3}+B_{2} B_{3} & A_{2} A_{4}+B_{2} B_{4} \\
A_{3} A_{1}+B_{3} B_{1} & A_{3} A_{2}+B_{3} B_{2} & A_{3}^{2}+B_{3}^{2} & A_{3} A_{4}+B_{3} B_{4} \\
A_{4} A_{1}+B_{4} B_{1} & A_{4} A_{2}+B_{4} B_{2} & A_{4} A_{3}+B_{4} B_{3} & A_{4}^{2}+B_{4}^{2}
\end{array}\right| \\
& =\left|\begin{array}{llll}
A_{1} & B_{1} & 0 & 0 \\
A_{2} & B_{2} & 0 & 0 \\
A_{3} & B_{3} & 0 & 0 \\
A_{4} & B_{4} & 0 & 0
\end{array}\right| \times\left|\begin{array}{llll}
A_{1} & A_{2} & A_{3} & A_{4} \\
B_{1} & B_{2} & B_{3} & B_{4} \\
0 & 0 & 0 & 0 \\
0 & 0 & 0 & 0
\end{array}\right|=0 .
\end{aligned}
$$

In proving the sufficiency of the conditions, we suppose that

$p_{1}, p_{2}, p_{3}, p_{4}$ are such that each three of the points is $d$-cyclic and $\Delta\left(p_{1}, p_{2}, p_{3}, p_{4}\right)=0$, and we show that there exist four points, say $p_{1}^{\prime}, p_{2}^{\prime}, p_{3}^{\prime}, p_{4}^{\prime}$ of the circle such that $p_{1}, p_{2}, p_{3}, p_{4} \approx p_{1}^{\prime}, p_{2}^{\prime}, p_{3}^{\prime}, p_{4}^{\prime} \cdot$. At least one of the angles $\alpha_{i j}$ is not equal to $T$, for if we suppose that each angle has the value. $T$, we find that $\Delta\left(p_{1}, p_{2}, p_{3}, p_{4}\right)$ equals - 16, which contradicts the hypothesis that $\Delta\left(p_{1}, p_{2}, p_{3}, p_{4}\right)=0$. We may assume the labeling. so that $\alpha_{12} \neq 0$. Hence $p, p_{2} \neq d$. By hypothesis there exist three points, say $p_{1}^{\prime}, p_{2}^{\prime}, p_{3}^{\prime}$, and three points, say $\bar{p}_{1}, \bar{p}_{2}, \bar{p}_{4}$, of the circle of metric diameter $d$ such that

$$
\begin{aligned}
& p_{1}, p_{2}, p_{3} \approx p_{1}^{\prime}, p_{2}^{\prime}, p_{3}^{\prime} \quad \text { and } \\
& p_{1}, p_{2}, p_{4} \approx \bar{p}_{1}, \bar{p}_{2}, \bar{p}_{4} .
\end{aligned}
$$

Thus $p_{1}^{\prime} p_{2}^{\prime}=p_{1} p_{2}=\bar{p}_{1} \bar{p}_{2} \neq d$.

Now we may make a congruent transfornation of the circle into 
itself so that $\bar{p}_{1}$ goes into the point $p_{1}^{\prime}$, and $\bar{p}_{2}$ goes into $p_{2}^{\prime}$. This transformation sends $\bar{p}_{4}$ into some point, say $p_{4}^{\prime}$, such that $p_{1}^{\prime} p_{4}^{\prime}=$ $\bar{p}_{1} \bar{p}_{4}=p_{1} p_{4}$ and $p_{2}^{\prime} p_{4}^{\prime}=\bar{p}_{2} \bar{p}_{4}=p_{2} p_{4}$. Since $p_{4}^{\prime}$ has its distances from two points, $p_{1}^{\prime}$ and $p_{2}^{\prime}$, determined and since these tro points are not diametral, the point $p_{4}^{\prime}$ is uniquely determined. We now have

$$
\begin{aligned}
& p_{1}, p_{2}, p_{3} \approx p_{1}^{\prime}, p_{2}^{\prime}, p_{3}^{\prime}, \\
& p_{1}, p_{2}, p_{4} \approx p_{1}^{\prime}, p_{2}^{\prime}, p_{4}^{\prime} .
\end{aligned}
$$

In order to show that the quadruple $p_{1}, p_{2}, p_{3}, p_{4}$ is congruent to the d-cyclic quadruple $p_{1}^{\prime}, p_{2}^{\prime}, p_{3}^{\prime}, p_{4}^{\prime}$, we have only to show that $p_{3} p_{4}=p_{3}^{\prime} p_{4}^{\prime}$. Fie define the expression $\Delta(x)$ as follows:

$$
\Delta(x)=\left|\begin{array}{cccc}
1 & \cos \alpha_{12} & \cos \alpha_{13} & \cos \alpha_{14} \\
\cos \alpha_{21} & 1 & \cos \alpha_{23} & \cos \alpha_{24} \\
\cos \alpha_{31} & \cos \alpha_{32} & 1 & \cos x \\
\cos \alpha_{41} & \cos \alpha_{42} & \cos x & 1
\end{array}\right|
$$

$\Delta(x)$ does not vanish identically since the coefficient of $\cos ^{2} x$, namely $-\left(1-\cos ^{2} \alpha / 2\right)$, does not ranish. By the necessity of the conditions of the theorem, a root of $\Delta(x)=0$ is $\frac{p_{3}^{\prime} p_{4}^{\prime}}{r}$, since $p_{1}^{\prime}, p_{2}^{\prime}, p_{3}^{\prime}, p_{4}^{\prime}$ are d-cyclic. By hypothesis, a root of $\Delta(x)=0$ is $\frac{p_{3} p_{A}}{2}$. Since $\Delta(x)=0$ is a quadratic equation in $\cos x, \Delta(x)=0$ has only two

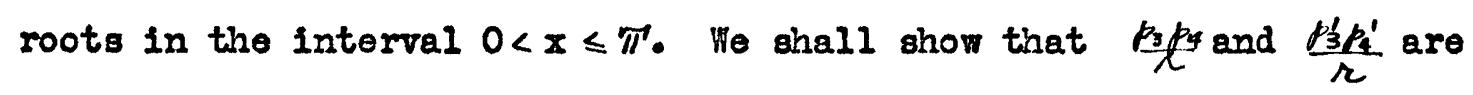
double roots. He may expand $\Delta(x)$ in the form *

$$
\Delta(x)=\frac{\Delta\left(p_{1}, p_{2}, p_{3}\right) \Delta\left(p_{1}, p_{2}, p_{4}\right)-\left|\begin{array}{ccc}
1 & \cos \alpha_{12} & \cos \alpha_{13} \\
\cos \alpha_{21} & 1 & \cos \alpha_{23} \\
\cos \alpha_{41} & \cos \alpha_{42} & \cos x
\end{array}\right|^{2}}{1-\cos ^{2} \alpha_{12}}
$$

* E. B. Stouffer, American Mathematical Monthly, Vol. 39 (1932), p. 165. 
By Theorem 1, $\Delta\left(p_{1}, p_{2}, p_{4}\right)$ and $\Delta\left(p_{1}, p_{2}, p_{4}\right)$ have the value 0 . Thus the equation $\Delta(x)=0$ may be written in the forn

$$
\left|\begin{array}{ccc}
1 & \cos \alpha_{12} & \cos \alpha_{13} \\
\cos \alpha_{21} & 1 & \cos \alpha_{23} \\
\cos \alpha_{41} & \cos \alpha_{42} & \cos x
\end{array}\right|^{2}=0 .
$$

Hence $\frac{p_{3} p_{4}}{\sim}$ and $\frac{p_{3} p_{4}}{\Omega}$ are double roots of the equation $\Delta(x)=0$. Therefore $p_{3} p_{4}=p_{3}^{\prime} p_{4}^{\prime}$ and the theorem is proved.

Theorems 1 and 3 characterize d-cyclic triples and quadruples. It has been shom that the circle has the congruence order four.* That 1s, a set is d-cyclic if each quadruple contained in the set is d-cyclic, Hence the necessary and sufficient condition that a set of points be d-cyclic is that each quadruple of the set satisfy the conditions stated in theorem $3 .+$

Te have defined a pseudo d-cyclic set of points as a set such that each three of the points is d-cyclic while the set itself is not d-cyclic. The remainder of this section is devoted to the characterization of such sets. First we shall characterize the pseudo d-cyclic quadruples. The three types of pseudo d-cyclic quadruples are the convex tripod, the pseudo-linear pseudo d-cyclic quadruple, and the proper pseudo d-cyclic quadruple, containing exactly three, exactly four, and no linear triples respectively.

Lemma. If $p_{1}, p_{2}, p_{3}, p_{4}$ form a convex tripod such that each triple is d-cyclic, then the sum of opposite distances equals $d$ and no

* Karl Menger, "New Foundations of Euclidean Geometry", American Joumal of Mathematics, Vol. 53 (1931), p. 725.

It is obvious that if $p_{1}, p_{2}, \ldots, p_{n}(n>4)$ are d-cyclic, then $\Delta\left(p_{1}, p_{2}, \ldots, p_{3}\right)=0$. Cf. Bocher, Introduction to Higher Algebra, p. 57. †L. M. Blumenthal, Loc. cit. 
two points are diametral.

From the definition of the convex tripod, we may assume the. labeling so that

$$
\begin{aligned}
& p_{2} p_{4}=p_{2} p_{1}+p_{1} p_{4} ; \\
& p_{3} p_{4}=p_{3} p_{1}+p_{1} p_{4} ; \\
& p_{2} p_{3}=p_{2} p_{1}+p_{1} p_{3} ; \\
& p_{2} p_{3}+p_{3} p_{1}+p_{2} p_{4}=2 d ; \text { where } p_{4} p_{3} \leq d .
\end{aligned}
$$

From these relations we obtain immediately

$$
\left.\begin{array}{r}
p_{1} p_{3}+p_{1} p_{2}+p_{1} p_{4}=d ; \\
p_{1} p_{3}=d-p_{2} p_{3} ; \\
p_{1} p_{2}=d-p_{3} p_{4} ; \\
p_{1} p_{4}=d-p_{2} p_{3}
\end{array}\right\}
$$

The relations (1) show that the sum of opposite distances equals $d$, and hence that no two of the points are diametral, since the points are distinct and consequently none of the distances is zero, which is what we wished to prove.

$$
\begin{aligned}
& \text { From (1) we have } \\
& \cos \alpha_{24}=-\cos \alpha_{13} ; \cos \alpha_{34}=-\cos \alpha_{12} ; \cos \alpha_{14}=-\cos \alpha_{23} ; \cos \alpha_{23}=\cos \left(\alpha_{12}+\alpha_{13}\right)
\end{aligned}
$$

Applying these relations, we may write

$$
\Delta\left(p_{1}, p_{2}, p_{3}, p_{4}\right)=\left|\begin{array}{cccc}
1 & \cos \alpha_{12} & \cos \alpha_{13} & \cos \alpha_{23} \\
\cos \alpha_{12} & 1 & \cos \alpha_{23} & \cos \alpha_{13} \\
\cos \alpha_{13} & \cos \alpha_{23} & 1 & \cos \alpha_{12} \\
\cos \alpha_{23} & \cos \alpha_{13} & \cos \alpha_{12} & 1
\end{array}\right| .
$$

If $p_{1}, p_{2}, p_{3}, p_{4}$ form a pseudo-linear pseudo d-cyclic quadruple, then opposite distances are equal*, and no tmo points are diametralt. We may assume the labeling so that

* Menger, Loc. cit., p. 744 .

$\ddagger$ L. M. Blumenthal, Loc. cit:, p. 730 . 


$$
\begin{aligned}
& p_{2} p_{3}=p_{1} p_{2}+p_{4} p_{3} ; p_{1} p_{4}=p_{1} p_{2}+p_{2} p_{4} ; \\
& p_{1} p_{4}=p_{1} p_{3}+p_{3} p_{4} ; p_{2} p_{3}=p_{2} p_{4}+p_{3} p_{4}
\end{aligned}
$$

Then $\quad p_{1} p_{2}=p_{3} p_{4} ; p_{1} p_{3}=p_{2} p_{4} ; p_{1} p_{4}=p_{2} p_{3}$. Hence the following relations exist;

$$
\cos \alpha_{24}=\cos \alpha_{13} ; \cos \alpha_{34}=\cos \alpha_{12} ; \cos \alpha_{14}=\cos \alpha_{23} ; \cos \alpha_{23}=\cos \left(\alpha_{12}+\alpha_{13}\right) \cdots
$$

Making use of these relations wo may again write $\Delta\left(p_{1}, p_{2}, p_{3}, p_{4}\right)$ in the form (I).

If $p_{1}, p_{2}, p_{3}, p_{4}$ form a proper pseudo d-cyclic quadruple, then "opposite distances are equal, no one of the distances equald the sum of the other two, the sum of three of the distances, no two of which are opposite, is equal to the length of the circle" and no tro of the points are diametral*. Thus $\alpha_{12}+\alpha_{23}+\alpha_{13}=2 \pi$, and hence $\cos \alpha_{23}=$ $\cos \left(\alpha_{12}+\alpha_{13}\right)$. We note that again in this case the relations (2) hold, and $\Delta\left(p_{1}, p_{2}, p_{3}, p_{4}\right)$ may be written in the form (I).

Surmarizing our investigations of the three kinds of pseudo $d$-cjclic quadruples, we find that if $p_{1}, p_{2}, p_{5}, p_{4}$ form a pseudo $d-$ cyclic quadruple of any of the three kinds, we may express $\triangle\left(p_{1}, p_{2}, p_{3}, p_{4}\right)$ in the form (I), where $\cos \alpha_{23}=\cos \left(\alpha_{12}+\alpha_{13}\right)$ and where none of the angles appearing in the determinant has the value $\mathbb{N}$. Expanding this determinant, we have

$$
\begin{aligned}
\Delta\left(p_{1}, p_{2}, p_{3}, p_{4}\right)= & {\left[\cos \alpha_{12}-\cos ^{3} \alpha_{12}-2 \cos \alpha_{12} \cos \alpha_{23}\right.} \\
& +\cos \alpha_{12} \cos ^{2} \alpha_{13} \\
& \left.+\cos \alpha_{12} \cos ^{2} \alpha_{23}\right]^{2} \\
= & {\left[\cos \alpha_{12}\left(1-\cos ^{2} \alpha_{12}-\cos ^{2} \alpha_{13}+\cos ^{2} \alpha_{12} \cos ^{2} \alpha_{13}+\sin ^{2} \alpha_{12} \sin ^{2} \alpha_{13}\right)\right.} \\
& \left.+2 \sin \alpha_{12} \sin \alpha_{13} \cos \alpha_{13}\left(1-\cos ^{2} \alpha_{12}\right)\right]^{2}
\end{aligned}
$$

* L. M. Blumenthal, "A Complete Characterization of d-Cyclic and Pseudo d-Cyclic Sets of Points", American Joumal of Mathematics, Vol. 54, p. 390. 


$$
\begin{aligned}
\Delta\left(p_{0}, p_{2}, p_{3}, p_{4}\right) & =-4 \sin ^{2} \alpha_{12} \sin ^{2} \alpha_{13}\left[\sin \alpha_{13} \cos \alpha_{12}+\sin \alpha_{12} \cos \alpha_{13}\right]^{2} \\
& =-4 \sin ^{2} \alpha_{12} \sin ^{2} \alpha_{13} \sin ^{2}\left(\alpha_{12}+\alpha_{23}\right) \\
& =-4 \sin ^{2} \alpha_{12} \sin ^{2} \alpha_{13} \sin ^{2} \alpha_{23}
\end{aligned}
$$

This value for $\Delta\left(p_{1}, p_{2}, p_{3}, p_{4}\right)$ is definitely negative, since no two of the points are diametral. Hence we have the following theorem:

Theorem 4. The determinant of a pseudo d-cyclic quadrupie is negative.

Applying the relation (3) and making use of theorems 1 and 3 , we have immediately the following theorem and corollaries.

Theorem 5. If all four third-order principal minors of the determinant $\Delta\left(p_{1}, p_{2}, p_{3}, p_{4}\right)$ vanish, and $0<\alpha_{i j}<\pi(1, i, 1,2,3,4)$, then $\Delta\left(p_{1}, p_{2} p_{2}, p_{4}\right)$ either vanishes or has the value $-4 \sin ^{2} \alpha_{12} \sin ^{2} \alpha_{12} \sin ^{2} \alpha_{23}$ where $\alpha_{12}<\pi$ and $\cos ^{2} \alpha_{12}=\cos ^{2} \alpha_{34}, \cos ^{2} \alpha_{23}=\cos ^{2} \alpha_{14}, \cos ^{2} \alpha_{13}=\cos ^{2} \alpha_{24}$.

Corollary 1. The points $p, p_{a}, p_{2} p_{4}$ form a pseudo d-cyclic convex tripod if and only if $\Delta\left(p_{1}, p_{2}, p_{3}, p_{4}\right) \neq 0$, each third-order principal minor vani ghes, and $\cos \alpha_{2}=-\cos \alpha_{34}, \cos \alpha_{23}=-\cos \alpha_{14}$, $\cos \alpha_{13}=-\cos \alpha_{24}$

Corollary 2. The points $p_{1}, p_{2}, p_{3}, p_{4}$ form a pseudo-linear nseude d-cyclic quadruple if and only if $\Delta\left(p_{1}, p_{2}, p_{3}, p_{4}\right) \neq 0$, each third-order principal minor vanishes, $\alpha_{12}=\alpha_{34}, \alpha_{23}=\alpha_{14}, \alpha_{12}=\alpha_{24}$, and all tripleg are linear.

Corollary 3. The points $p_{1}, p_{2}, p_{3}, p_{4}$ form a proper pseudo derclic quedmple if and only if $\Delta\left(p_{1}, p_{2}, p_{3}, p_{4}\right) \neq 0$, each third-order principal minor vanishos, $\alpha_{12}=\alpha_{34}, \alpha_{23}=\alpha_{14}, \alpha_{13}=\alpha_{24}$, and no triples are linear.

We shall now describe methods of construeting the three forms of pseudo d-cyclic quadruples. 
The pseudo d-cyclic convex tripod and the proper pseudo d-cyclis quadruple. Let $p_{1}^{\prime}, p_{2}^{\prime}, p_{3}^{\prime}$ be three non-linear points of the circle of

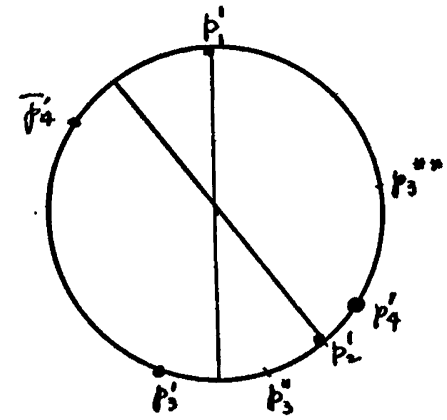

Fig. 1. metric diameter d. Reflect $p_{3}^{\prime}$ in the diameter through $p_{l}^{\prime}$, obtaining the point $p_{3}^{*}$. Reflect $p_{3}^{\prime}$ in the diameter through $\mathrm{p}_{2}^{\prime}$, obtaining $\mathrm{p}_{3}^{* * *}$. Let $\mathrm{p}_{4}^{\prime}$ and $\bar{p}_{4}^{\prime}$ be the two points of the circle equidistant from $p_{3}^{*}$ and $p_{3}^{* *}$.

We shall let $p_{4}^{\prime}$ be the midpoint of the shorter arc joining $p_{3}^{*}$ and $p_{3}^{* *}$, and $\bar{p}_{4}^{\prime}$ the point diametral to $p_{4}^{\prime}$ (see Fig.1). Define the quadruple $p_{1}, p_{2}, p_{3}, p_{4}$ such that $p_{1} p_{2}=p_{1}^{\prime} p_{2}^{\prime} ; p_{1} p_{3}=p_{1}^{\prime} p_{3}^{\prime} ; p_{2} p_{3}=p_{2}^{\prime} p_{3}^{\prime} ;$ $p, p_{4}=p_{1}^{\prime} p_{4}^{\prime} ; p_{2} p_{4}=p_{2}^{\prime} p_{4}^{\prime} ; p_{3} p_{4}=p_{3}^{*} p_{4}^{\prime}=p_{3}^{* *} p_{4}^{\prime}$. The triples $p_{1}, p_{2}, p_{4} ;$ $p_{1}, p_{3}, p_{4} ; p_{2}, p_{3}, p_{4}$ are $d$-cyclic and linear with $p_{4}$ between $p_{1}$ and $p_{2}$, $p_{1}$ and $p_{3}, p_{2}$ and $p_{3} \cdot$ The triple $p_{1}, p_{2}, p_{3}$ is not linear, but is d-cyclic. Hence, $p_{1}, p_{2}, p_{3}, p_{4}$ form a pseudo d-cyclic convex tripod.

If $p_{1}, p_{2}, p_{3}, p_{4}$ are defined such that $p_{1} p_{2}=p_{1}^{\prime} p_{2}^{\prime}, p_{1} p_{3}=p_{1}^{\prime} p_{3}^{\prime}$, $p_{2} p_{3}=p_{2}^{\prime} p_{3}^{\prime}, p_{1} p_{4}=p_{1}^{\prime} \bar{p}_{4}^{\prime}, p_{2} p_{4}=p_{2}^{\prime} p_{4}^{\prime}, p_{3} p_{4}=p_{3}^{*} \bar{p}_{4}^{\prime}=p_{3}^{* * p_{4}^{\prime}}$, then it is seen immediately that each triple is d-cyclic and not linear and that opposite distances are equal. In this case $p,, p_{2}, p_{3}, p_{4}$ form a proper pseudo d-cyclic quadruple. Provided that $p_{1}^{1}, p_{2}^{1}, p_{3}^{1}$ are not linear, it is true for any arrangement of the points that the convex tripod and the proper pseudo d-cyclic quadruple can be constructed by the methods described above. One of the points $p_{4}^{\prime}$ and $\bar{p}_{4}^{\prime}$-which one depending upon the positions of $p_{1}^{\prime}, p_{2}^{\prime}, p_{3}^{\prime}$ - will give the convex tripod and the other will give the proper pseudo d-cyclic quadruple. By choosing the points $p_{1}^{\prime}, P_{2}^{\prime}, P_{3}^{\prime}$ such that the triple is equilateral and by applying the methods described above, a proper pseudo d-cyclic quadruple which is equilateral may be constructed. 
The pseudo-linear pseudo d-cyclic quadmuple. If the points $p_{1}^{1}, p_{2}^{\prime}, p_{3}^{\prime}$ are chosen so that the triple is linear and no two of the points are diametral and if $\mathrm{p}_{4}^{\prime}$ and $\overline{\mathrm{p}}_{4}^{\prime}$ are determined as in the previous case and the quadruple $p_{1}, p_{2}, p_{3}, p_{4}$ defined as above, then in either case each triple is d-cyclic and linear, opposite distances are equal, and the four points form a pseudo-linear pseudo d-cyclic quadruple.

Pseudo d-cyclic sets no four points of which form a convex tripod are called regular. In order to establish the theorem characterizing sets of this type which contain more than four points we shall prove two lemas concerning the fifth-order determinant $\Delta\left(p_{1}, p_{2}, p_{3}, p_{4}, p_{5}\right)$. Consider the determinant $\Delta\left(p_{1}, p_{2}, p_{3}, p_{4}, p_{5}\right)$ subject to the following hypotheses:

(a). $0<\alpha_{i j} \leq \pi$

(b). Each third-order principal minor vanishes.

(c). At least one fourth-order principal minor does not vanish.

(d). If $\Delta\left(p_{i}, p_{j}, p_{k}, p_{l}\right)$ is any non-vanishing fourth-order principal minor, it is possible so to arrange the labeling of the elements of the minor that $\cos \alpha_{i j}+\cos \alpha_{k l} \neq 0$.

Lemme 1. $\Delta\left(p_{1}, p_{2}, p_{1}, p_{9}, p_{r}\right)$ does not contain exactly one non-vaniahing fourth-order principal minor.

We shall assume that $\Delta\left(p_{1}, p_{2}, p_{3}, p_{4}\right) \neq 0$. Then by theorem 5 , $\Delta\left(p_{1}, p_{2}, p_{3}, p_{4}\right)$ is negative and none of the six angles contained in this minor has the value $\pi$. By the three corollaries to theorem 5 we have

$$
\cos \alpha_{12}=\cos \alpha_{34} ; \cos \alpha_{13}=\cos \alpha_{24} ; \cos \alpha_{23}=\cos \alpha_{14} \text {. }
$$

We suppose that the four remaining fourth-order principal minors

$$
\Delta\left(p_{1}, p_{2}, p_{3}, p_{5}\right), \Delta\left(p_{1}, p_{2}, p_{4}, p_{5}\right), \Delta\left(p, p_{3}, p_{4}, p_{5}\right), \text { and } \Delta\left(p_{2}, p_{3}, p_{4}, p_{5}\right)
$$


$-14-$

all vanish and show that this assumption leads to a contradiction.

Expanding each of these four minors, we obtain the following relations.

$$
\begin{aligned}
& \left|\begin{array}{ccc}
1 & \cos \alpha_{12} & \cos \alpha_{13} \\
\cos \alpha_{12} & 1 & \cos \alpha_{23} \\
\cos \alpha_{15} & \cos \alpha_{25} & \cos \alpha_{35}
\end{array}\right|=0 ;\left|\begin{array}{ccc}
1 & \cos \alpha_{12} & \cos \alpha_{14} \\
\cos \alpha_{12} & 1 & \cos \alpha_{24} \\
\cos \alpha_{15} & \cos \alpha_{25} & \cos \alpha_{45} \\
1 & \cos \alpha_{13} & \cos \alpha_{14} \\
\cos \alpha_{15} & 1 & \cos \alpha_{34} \\
\cos \alpha_{15} & \cos \alpha_{35} & \cos \alpha_{45}
\end{array}\right|=0 ;\left|\begin{array}{ccc}
1 & \cos \alpha_{23} & \cos \alpha_{24} \\
\cos \alpha_{23} & 1 & \cos \alpha_{34} \\
\cos \alpha_{25} & \cos \alpha_{35} & \cos \alpha_{45}
\end{array}\right|=0 ;
\end{aligned}
$$

Writing $a=\cos \alpha_{12}=\cos \alpha_{34} ; b=\cos \alpha_{13}=\cos \alpha_{24} ; c=\cos \alpha_{23}=\cos \alpha_{14}$,

and expanding the four determinants above, we have

$(a c-b) \cos \alpha_{15}+(a b-c) \cos \alpha_{25}+\left(1-a^{2}\right) \cos \alpha_{35}$

$=0$

$(a b-c) \cos \alpha_{15}+(a c-b) \cos \alpha_{25}$

$$
+\left(1-a^{2}\right) \cos \alpha_{45}=0
$$

$(a b-c) \cos \alpha_{15}$

$$
\begin{aligned}
+(b-c-a) \cos \alpha_{35}+\left(1-b^{2}\right) \cos \alpha_{45} & =0 \\
(a c-b) \cos \alpha_{45}+(b-a) \cos \alpha_{35}+\left(1-c^{2}\right) \cos \alpha_{45} & =0
\end{aligned}
$$

Now $\cos \alpha_{15}, \cos \alpha_{25}, \cos \alpha_{35}, \cos \alpha_{45}$ ere not all zero. In fact, wo get a contradiction by supposing that two of them, say $\cos \alpha_{15}$ and $\cos \alpha_{25}$, are zero. For if so, then $\alpha_{15}=\alpha_{25}=\frac{\pi}{2}$. Therefore, since $\Delta\left(p_{1}, p_{2}, p_{5}\right)=0, \alpha_{12}$ must have the value 0 or $\pi$, which is impossible. The four equations cannot be satisfied, then, unless

$$
\left|\begin{array}{cccc}
a c-b & a b-c & 1-a^{2} & 0 \\
a b-c & a c-b & 0 & 1-a^{2} \\
a b-e & 0 & b e-a & 1-b^{2} \\
0 & a c-b & b e-a & 1-c^{2}
\end{array}\right|=0
$$

That is

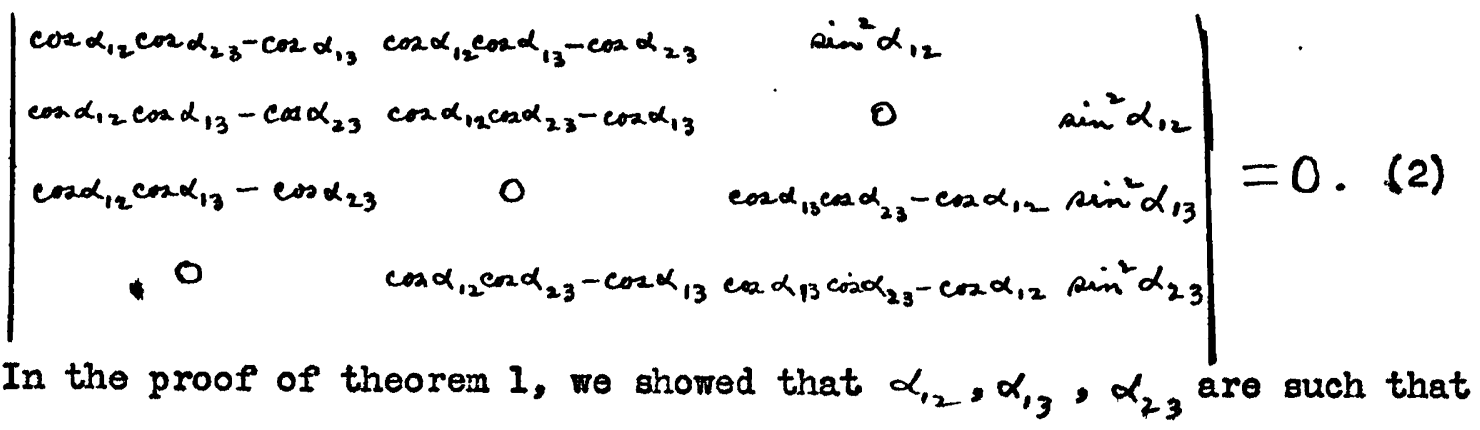


either $\alpha_{12}+\alpha_{13}+\alpha_{23}=2 \pi$ or one angle equals the sum of the other two. Thus, two cases present themselves.

Case 1. $\quad \alpha_{12}+\alpha_{13}+\alpha_{23}=2 \pi$

In this case equation (2) becomes

$$
\left|\begin{array}{cccc}
\sin \alpha_{12} \sin \alpha_{23} & \sin \alpha_{12} \sin \alpha_{13} & \sin ^{2} \alpha_{12} & 0 \\
\sin \alpha_{12} \sin \alpha_{13} & \sin \alpha_{12} \sin \alpha_{23} & 0 & \sin ^{2} \alpha_{12} \\
\sin \alpha_{12} \sin \alpha_{13} & 0 & \sin \alpha_{13} \sin \alpha_{23} & \sin ^{2} \alpha_{13} \\
0 & \sin \alpha_{12} \sin \alpha_{23} & \sin \alpha_{13} \sin \alpha_{23} & \sin ^{2} \alpha_{23}
\end{array}\right|=0
$$

Factoring sin $\alpha_{12}$ out of the first two rows, sin $\alpha_{13}$ out of the third row, and sin $\alpha_{23}$ out of the fourth row, and interchanging the first and fourth colums and the second and third colurms, we have

$$
\left|\begin{array}{cccc}
0 & \sin \alpha_{12} & \sin \alpha_{13} & \sin \alpha_{23} \\
\sin \alpha_{12} & 0 & \sin \alpha_{23} & \sin \alpha_{13} \\
\sin \alpha_{13} & \sin \alpha_{23} & 0 & \sin \alpha_{12} \\
\sin \alpha_{23} & \sin \alpha_{13} & \sin \alpha_{12} & 0
\end{array}\right|=0 .
$$

Evaluation of this determinant gives the relation

$$
\begin{aligned}
& \left(\sin \alpha_{12}+\sin \alpha_{23}+\sin \alpha_{13}\right)\left(\sin \alpha_{12}+\sin \alpha_{23}-\sin \alpha_{13}\right) \\
& \quad x\left(\sin \alpha_{12}-\sin \alpha_{23}+\sin \alpha_{13}\right)\left(-\sin \alpha_{12}+\sin \alpha_{23}+\sin \alpha_{13}\right)=0 .
\end{aligned}
$$

The first factor cannot vanish. Hence one of the remaining factors vanishes. Suppose the second factor ranishes. Then

$$
\text { From (3) } \quad \sin \alpha_{13}+\sin \alpha_{12} \cos \alpha_{23}+\cos \alpha_{12} \sin \alpha_{23}=0
$$

Adding these two equations we have

$$
\sin \alpha_{12}\left(1+\cos \alpha_{23}\right)+\sin \alpha_{23}\left(1+\cos \alpha_{12}\right)=0 \text {. }
$$

This is impossible since both terms are definitely positive. Similarly we may show it is impossible that any of the remaining factors vanish. . 
Case 2. One of the angles $\alpha_{12}, \alpha_{13}, \alpha_{23}$ equals the sum of the other two. We may assume that

$$
\alpha_{13}=\alpha_{12}+\alpha_{23} \text {. }
$$

In this case, equation (2) becomes

$$
\left|\begin{array}{cccc}
\sin \alpha_{12} \sin \alpha_{23} & -\sin \alpha_{12} \sin \alpha_{13} & \sin ^{2} \alpha_{12} & 0 \\
-\sin \alpha_{12} \sin \alpha_{13} & \sin \alpha_{12} \sin \alpha_{23} & 0 & \sin ^{2} \alpha_{12} \\
-\sin \alpha_{12} \sin \alpha_{13} & 0 & -\sin \alpha_{13} \sin \alpha_{23} & \sin ^{2} \alpha_{13} \\
0 & \sin \alpha_{12} \sin \alpha_{23} & -\sin \alpha_{13} \sin \alpha_{23} & \sin ^{2} \alpha_{23}
\end{array}\right|=0
$$

Factoring sin $\alpha_{12}$ out of the first two rows, sin $\alpha_{13}$ out of the third row, and $\sin \alpha_{2}$, out of the fourth row, and interchanging the first and fourth columns and the second and third columns, we have

$$
\left|\begin{array}{cccc}
0 & \sin \alpha_{12} & -\sin \alpha_{13} & \sin \alpha_{23} \\
\sin \alpha_{12} & 0 & \sin \alpha_{23} & -\sin \alpha_{13} \\
\sin \alpha_{13} & -\sin \alpha_{23} & 0 & -\sin \alpha_{12} \\
\sin \alpha_{23} & -\sin \alpha_{13} & \sin \alpha_{12} & 0
\end{array}\right|=0
$$

This determinant is the same as the corresponding determinant considered In Case 1 except for the factor -1 , as may be seen by multiplying the second and fourth columns and the first row by -1 . Hence, again we have the equation (4). Assume first that the second factor of (4) vanishes.

Then

$$
\sin \alpha_{12}+\sin \alpha_{23}-\sin \alpha_{13}=0 .
$$

From (5)

$$
\sin \alpha_{12} \cos \alpha_{23}+\sin \alpha_{23} \cos \alpha_{12}-\sin \alpha_{13}=0
$$

Subtracting these two equations we have

$$
\sin \alpha_{12}\left(1-\cos \alpha_{23}\right)+\sin \alpha_{23}\left(1-\cos \alpha_{12}\right)=0 \text {. }
$$

This is impossible, since both terms are definitely positive. Assuming the third factor of (4) vanishes we have

From (5)

$$
\sin \alpha_{13}+\sin \alpha_{12}-\sin \alpha_{23}=0 .
$$

$$
\sin \alpha_{13} \cos \alpha_{12}-\sin \alpha_{12} \cos \alpha_{13}-\sin \alpha_{23}=0 \text {. }
$$


Subtracting these two equations, we obtain the relation

$$
\sin \alpha_{12}\left(1+\cos \alpha_{13}\right)+\sin \alpha_{13}\left(1-\cos \alpha_{12}\right)=0 \text {. }
$$

which is also impossible. Similarly we may show that it is impossible for the fourth factor of (4) to vanish. Hence the proof of the lemma is comploted.

Lemme 2. No fourth-order principal minor of $\Delta\left(p_{1}, p_{2}, p_{3}, p_{4}, p_{5}\right)-$ vanishes.

By the preceding lemma, there are at least two non-vaniahing fourth-order principal minors. We may assume, them, that $\Delta\left(p_{1}, p_{2}, p_{3}, p_{4}\right)$ and $\Delta\left(p_{1}, p_{2}, p_{3}, p_{5}\right)$ do not ranish. Then none of the nine angles contained in these two minors has the value $\pi$, and

$$
\left.\begin{array}{l}
\cos \alpha_{12}=\cos \alpha_{34}=\cos \alpha_{35} \\
\cos \alpha_{13}=\cos \alpha_{24}=\cos \alpha_{25} \\
\cos \alpha_{23}=\cos \alpha_{14}=\cos \alpha_{15}
\end{array}\right\} .
$$

by the corollaries to theorem 5 .

Suppose that any other fourth-order principal minor, say $\Delta\left(p_{1}, p_{2}, p_{4}, p_{5}\right)$ vanishes. Then from the expansion of $\Delta\left(p_{1}, p_{2}, p_{4}, p_{5}\right)$ we have

$$
\left|\begin{array}{ccc}
1 & \cos \alpha_{12} & \cos \alpha_{14} \\
\cos \alpha_{12} & 1 & \cos \alpha_{24} \\
\cos \alpha_{15} & \cos \alpha_{25} & \cos \alpha_{45}
\end{array}\right|=0
$$

Applying (1), we may write this equation in the form

$$
\left|\begin{array}{ccc}
1 & \cos \alpha_{12} & \cos \alpha_{14} \\
\cos \alpha_{12} & 1 & \cos \alpha_{24} \\
\cos \alpha_{14} & \cos \alpha_{24} & \cos \alpha_{45}
\end{array}\right|=0 .
$$

Consider the function $\varphi(x)$ defined as follows:

$$
\varphi(x)=\left|\begin{array}{ccc}
1 & \cos \alpha_{12} & \cos \alpha_{14} \\
\cos \alpha_{12} & 1 & \cos \alpha_{24} \\
\cos \alpha_{14} & \cos \alpha_{24} & x \\
\cdot & &
\end{array}\right|
$$


Since $\Delta\left(p_{1}, p_{2}, p_{4}\right)=0$, one root of $\varphi(x)=0$ is $x=1$ : Noreover, since $\varphi(x)=0$ is a linear equation and since the coefficient of $x$, namely $\left(.1-\cos ^{2} \alpha_{12}\right)$ does not vanish, 1 is the only root of $P(x)=0$. Therefore $\cos \alpha_{45}=1$, and hence $\alpha_{46}=0$, which is impossible. Hence the lemma is proved.

Hence, under the conditions imposed, no fourth-order principal minor of the determinant $\Delta\left(p_{1}, p_{2}, p_{3}, p_{4}, p_{5}\right)$ vanishes. By theorem 3 each fourth-order principal minor is negative and, by the corollaries to theorem 5, opposite angles in each quadruple are equal. Making use of this fact, we have immediately the following theorem.

Theorem 6. Each element of the determinant $\Delta\left(p_{1}, p_{2}, p_{3}, p_{4}, p_{5}\right)$ outbide the principal diagonal has the same value, and this value is $-\frac{1}{3}$.

The first part of this theorem is obtained by equating opposite angles in each quadruple. Since each triple is d-cyclic, in order that each of the three angles in each triple have the same value, this value must be $\frac{2 \pi}{3}$. Hence $\cos \alpha_{i j}=-\frac{1}{2}$. The value of this determinant is found to be $-\left(\frac{3}{2}\right)^{4}$.

Stating theorem 6 as a theorem on sets of points, wa have the Theorem 7. A regular pseudo d-cyclic quintuple is equilateral*. By applying mathematical induction we obtain a more general theorem Theorem 8. A regular pseudo d-cyclic set containing more than four points is equilateral.

We have shown that the theorem holds if the set consiats of exactly five points. We shall assume the theorem holds for sets containing $k$ points $(k>4)$ and show that it follows that the theorem is true

* This theorem as well as the following one are proved by L. M. Blumenthal in the paper "Concerming Regular Pseudo d-Cyclic Sets", American Journal of Mathematics, Vol. 54, pp. 729-738. The proof given above for theorem7 is quite different from the proof given by Blumenthal. 
for sets containing $k+1$ points.

Let $p_{1}, p_{2}, \ldots, p_{k}, p_{k+1}$ be a regular psoudo d-cjclic set containing exactly $k+1$ points. At least one $k$-tuple contained in these $k+1$ points is a pseudo d-cyclic set, for otherwise each $k$ points and hence each four points is d-cyclic. But since the circle has the congruence order four, if each four points is $d$-cyclic, the $k+1$ points are $d$-cyclic, which contradicts the hypothesis that the set is pseudo d-cyclie. Me may assume the labeling, then, so that $p_{1}, p_{2}, \ldots, p_{k}$ are pseudo d-cyclic. At least one other $k$-tuple is preudo d-cyclic. For if re suppose that all k-tuples except $p, p_{2}, \ldots, p_{k}$ are $d$-cyclic, then each quadruple contained in them is d-cyclic. But all the quadruples contained in $\mathrm{p}_{1}, \mathrm{p}_{2}, \ldots, \mathrm{p}_{f}$ are contained in the $\mathrm{k}$ remaining $\mathrm{k}$-tuples. Hence, from our supposition it follows that the set $p_{1}, p_{2}, \ldots, p_{k}$ is $d$-cyclic, which is impossible. Thus, we may assume that $p_{2}, p_{3}, \ldots, p_{k+1}$ is a pseudo d-cyclic k-tuple. Since we assume the theorem true for pseudo $d$-cyclic sets containing $k$ points, the sets $p_{1}, p_{2}, \ldots, p_{k}$ and $p_{2}, p_{3}, \ldots$, $P_{k}, p_{k+1}$ gre equilateral and each distance contained in the tro sets equals $\frac{2 d}{3}$. All the distances, then, except $p, p_{k+1}$ have been. shown to have the value $\frac{2 d}{3}$ - To show that $p_{1} p_{k+1}$ equals $\frac{2 d}{3}$, we consider the triple $p_{1}, p_{2}, p_{k+1} \cdot$ This triple is d-cyclic and

$$
p_{1} p_{2}=p_{2} p_{k+1}=\frac{2 d}{3}
$$

Evidently the triple $p_{1}, p_{2}, p_{k+1}$ cannot be linear. Hence

$$
p_{1} p_{2}+p_{2} p_{R+1}+p_{1} p_{k+1}=2 d
$$

Therefore $p_{1} p_{k+1}=\frac{2 d}{3}$, and the theorem is proved.

Changing back to our determinantal notation, we have the following theorem as the analogue to theorem 8. 
Theorem 9. If $\Delta\left(p_{1}, p_{2}, \ldots, p_{n}\right), n>4$, is such that

(a). $0<\alpha_{i j} \leq \pi$.

(b). Each third-order principal minor vanishes.

(c). At least one fourth-order principal minor does not

vanish.

(d). If $\Delta\left(p_{i}, p_{j}, p_{f}, p_{f}\right)$ is any non-vanishing fourthorder principal minor, it is possible so to arrange the labeling of the elements of the minor that

\section{$\cos \alpha_{i j}+\cos \alpha_{k b} \neq 0$}

then each angle contained in the determinant has the value $\frac{2 \pi}{3}$, and the determinant has the value $-\frac{1}{2}\left(\frac{3}{2}\right)^{n-1}(n-3)$.

In order to obtain the value of $\Delta\left(p_{1}, p_{2}, \ldots, p_{n}\right)$ given above, we evaluate the determinant of the n-th order defined as follows.

$$
\begin{aligned}
& A_{n}=\left|\begin{array}{cccccc}
1 & a & a & a & \ldots & a \\
a & 1 & a & a & \ldots & a \\
a & a & 1 & a & \ldots & a \\
a & a & a & 1 & \cdots & a \\
\vdots & \vdots & \vdots & \vdots & & \vdots \\
a & a & a & a & \ldots & 1
\end{array}\right|=\left|\begin{array}{cccccc}
1-a & a-1 & 0 & 0 & \ldots & 0 \\
a & 1 & a & a & \ldots & a \\
0 & a-1 & 1-a & 0 & \ldots & 0 \\
0 & a-1 & 0 & 1-a & \ldots & 0 \\
\vdots & \vdots & \vdots & \vdots & \vdots \\
0 & a-1 & 0 & 0 & \ldots & \vdots
\end{array}\right| \\
& =(1-a)\left|\begin{array}{ccccc}
1 & a & a & \cdots & a \\
a-1 & 1-a & 0 & \cdots & 0 \\
a-1 & 0 & 1-a & \cdots & 0 \\
\vdots & \vdots & \vdots & & \vdots \\
a-1 & 0 & 0 & \cdots & \vdots-a
\end{array}\right|-\left|\begin{array}{ccccc}
a-1 & 0 & 0 & \cdots & 0 \\
a-1 & 1-a & 0 & \cdots & 0 \\
a-1 & 0 & 1-a & \cdots & 0 \\
\vdots & \vdots & \vdots & & \vdots \\
a-1 & 0 & 0 & \cdots & i-a
\end{array}\right| \\
& =(1-a) A_{n-1}+a(1-a)^{n-1}
\end{aligned}
$$

Since $A_{1}=1$, we have 


$$
\begin{aligned}
& A_{1}=1 \\
& A_{2}=(1-a)(1+a) \\
& A_{3}=(1-a)^{2}(1+2 a)
\end{aligned}
$$

which suggest the formula

$$
A_{n} \quad(1-a)^{n-1}[1+(n-1) a]
$$

If we assume the formula (2) holds for $n=k$, then by applying (1) we find that the formula holds for $n=k+1$. Hence, by mathematical Induction, the formula (2) is correct. If we substitute $-\frac{1}{2}$ for $a$ in (2) we obtain the value $-\frac{1}{2}\left(\frac{3}{2}\right)^{n-1}(n-3)$ for $A_{x}$ which is what we wished to show.

\section{The Sphere, $\underline{S}_{2, r}$.}

Te shall characterize $r$-spheric and pseudo $r$-spheric sets by means of theorems similar to the theorems characterizing d-cyclic and pseudo d-cyclic sets. It is evident that d-cyclic sets are included among the $r$-spheric sets. Throughout this section $d$ equals $\pi r$.

We define the angle $\alpha_{i j}$ and the determinant $\Delta\left(p_{1}, p_{2}, \ldots, p_{n}\right)$ as in Section I. We shall denote by 0 the center of the sphere of radius $r$ and by $r\left(p_{1}, p_{2}, \ldots, p_{n}, 0\right)$ the volume of the simplex determined by the $n+1$ points $p_{1}, p_{2}, \ldots, p_{n}, 0$.

Lempe 1. $\Delta\left(p_{1}, p_{2}, \ldots, p_{n}\right)=\left(\frac{n !}{r^{n}}\right)^{2} v^{2}\left(p_{1}, p_{2}, \ldots, p_{1}, 0\right)$.

Denoting the Euclidean diatance between $p_{i}$ and $p_{j}$ by $\overline{i j}$ wo have

$$
\begin{gathered}
\overline{i j}=2 x^{2}-2 x^{2} \cos \alpha_{i j} \\
\nabla^{2}\left(p_{1}, p_{2}, \ldots, p_{n}, 0\right)=\frac{(-1)^{n+1}}{(n !)^{2} 2^{n}} D\left(p_{1}, p_{n}, \ldots, p_{n}, 0\right) *
\end{gathered}
$$

But 


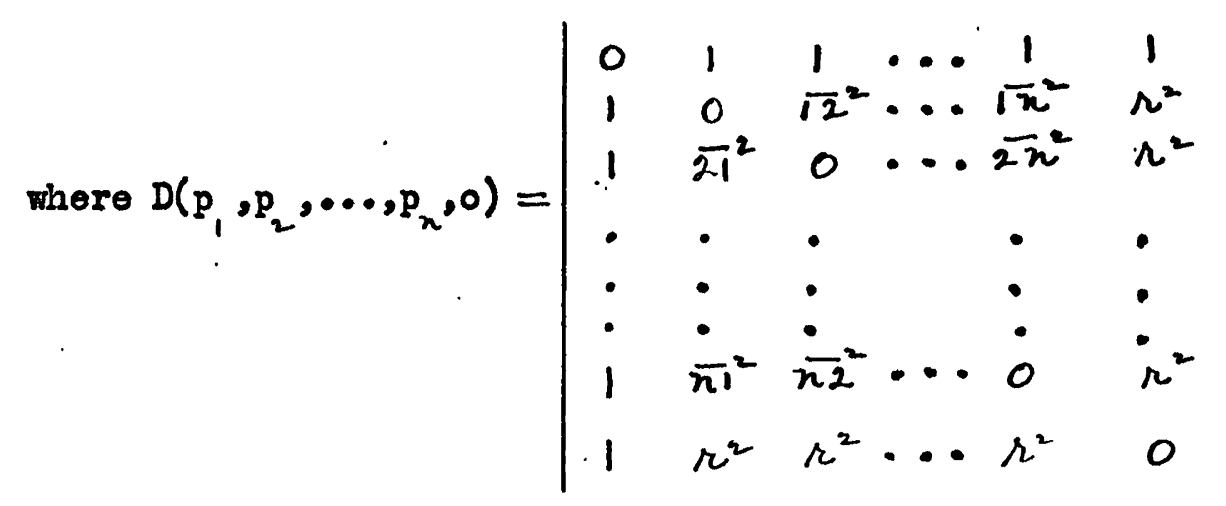

Subtracting the last row of the determinant $D\left(p_{1}, p_{2}, \ldots, p_{n}, 0\right)$ from each preceding row except the first, and subtracting the last column from each preceding column, we have

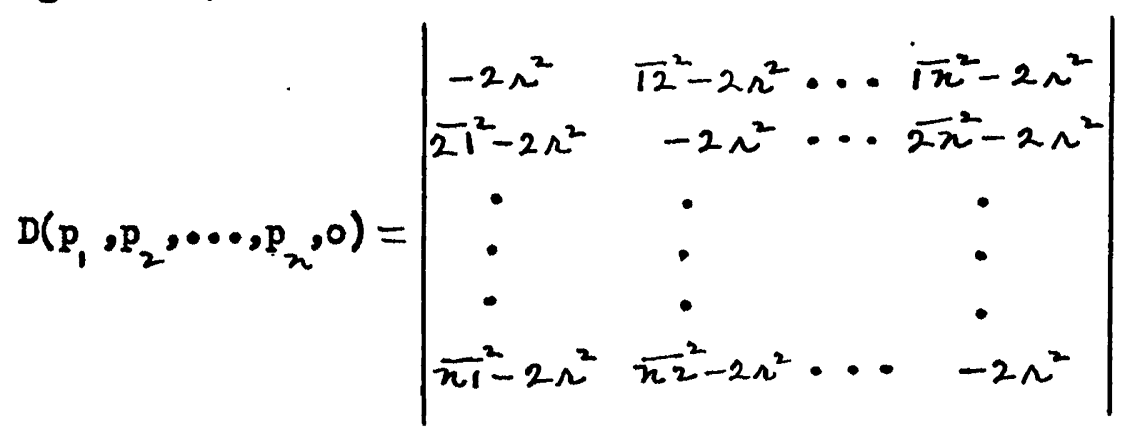

Applying (1) this equation may bo written in the form

$$
D\left(p_{1}, p_{2}, \ldots, p_{n}, 0\right)=(-1)^{n+1}\left(2 n^{2}\right)^{n} \Delta\left(p_{1}, p_{2}, \ldots, p_{n}\right) \cdot
$$

Substituting this value for $D\left(p_{1}, p_{2}, \ldots, p_{n}, 0\right)$ in (2) we obtain the desired relation

$$
\Delta\left(p_{1}, p_{2}, \ldots, p_{n}\right)=\left(\frac{\pi !}{r^{n}}\right)^{2} v^{2}\left(p_{1}, p_{2}, \ldots, p_{n}, 0\right)
$$

As a corollary to this lemma we have the following lema.

Lemma 2. If $p_{2} p_{2} \ldots, p_{2}(n>3)$ are $r$-spheric, then

$\Delta\left(p_{1}, p_{2}, \ldots, p_{2}\right)=0$

If $n$ equals 3 , them $v^{2}\left(p_{1}, p_{2}, p_{3}, 0\right)$ has the value zero if $p_{1}$, $p_{2}, p_{3}, 0$ lie in a plane and is politive if $p_{1}, p_{2}, p_{3}, 0$ do not lie in a plane. Since $\dot{p}_{1}, p_{2}, p_{3}, 0$ lie in a plane if and only if $p_{1}, p_{2}, p_{3} 11 \theta$ on a great circle of the sphere, we have the following lemma.

Lemma 3. If $p_{1}, p_{2}, p_{3}$ are $r$-spheric and not d-cyclic then 
Theorem 10. Three points $p_{1}, p_{2}, p_{3}$ of a semi-metric space are r-spheric if and only if $0<\alpha_{i j} \leqslant \pi(i, j=1,2,3)$ and $\Delta\left(p_{1}, p_{2}, p_{3}\right) \geqslant 0$.

The necessity of the conditions follows immediately from Lemma 3 and theorem 1. To prove the suffieiency of the conditions we consider two cases.

Case 1. $\Delta\left(p_{1}, p_{2}, p_{2}\right)=0$. In this case the three pointe are d-cyclic, by theorem 1, and hence are congruent to three points of a great circle of the sphere.

Gase 2. $\Delta\left(p_{1}, p_{2}, p_{3}\right)>0$. We shall show that the three angles $\alpha_{12}, \alpha_{13}, \alpha_{23}$ satisfy the triangular inequality and that their sum is less than $2 \pi$. We have

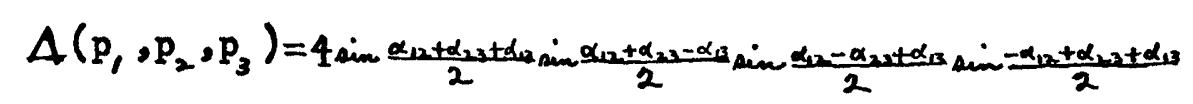

Each of the three angles cannot have the value $\pi$, for if so then $\Delta\left(p_{1}, p_{2}, p_{3}\right)$ has the value -4 , which is impossible. Hence

$$
0<\alpha_{12}+\alpha_{23}+\alpha_{13}<3 \pi \text {. }
$$

We shall show first that

$$
0<\alpha_{12}+\alpha_{23}+\alpha_{13}<2 \pi
$$

Obviously $\alpha_{12}+\alpha_{23}+\alpha_{13} \neq 2 \pi$ for if so, then by (1) $\Delta\left(p_{1}, p_{2}, p_{3}\right)$ equals zero which is impossible. Te suppose, then, that

$$
2 \pi<\alpha_{12}+\alpha_{23}+\alpha_{13}<3 \pi
$$

and show that this supposition leads to a contradiction. We shall

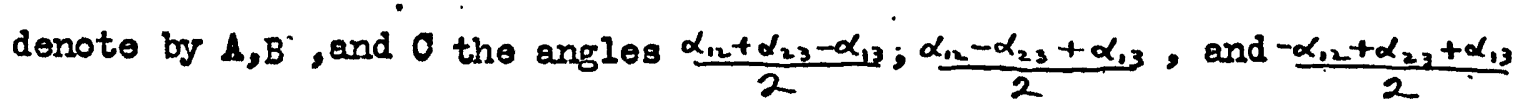
respectively. Then $|A|,|B|$, and $|C|$ are all less than $\pi$. Nor, no one of the angles $A, B, C$ is negative; for suppose that any one of them, say $A$, is negative. Then $\alpha_{12}+\alpha_{23}<\alpha_{13} \leq \pi$. Hence $\alpha_{12}+\alpha_{23}+\alpha_{13} \leq 2 \pi$ which contradicts (2). Therefore sin $A$, sin $B, \sin C$ are all positive. From (2), $\sin \frac{\alpha_{12}+\alpha_{23}+\alpha_{13}}{2}$ is negative. Substituting in (1), then, we find that $\Delta\left(p, p_{2}, p_{3}\right)$ is negative and we have a contradiction. 
Hence

$$
0<\alpha_{12}+\alpha_{23}+\alpha_{13}<2 \pi
$$

Therefore sin $\frac{\alpha_{12}+\alpha_{23}+\alpha_{13}}{2}$ is positive. Since $\Delta\left(p_{1}, p_{2}, p_{3}\right)$ is positive, we seo from (1) that $\sin A \sin B$ sin $C$ must be positive. Hence, either all three of the factors $\sin A, \sin B$, and $\sin C$ are positive, or two of them are negative and one is positive. We show that the latter condition cannot arise. Suppose that two of the factors, say sin A and sin $B$, are negative. Then since $|A|$ and $|B|$ are less than $\pi, A$ and $B$ themselves must be negative. Hence, we have

$$
\begin{aligned}
& \alpha_{12}+\alpha_{23}-\alpha_{13}<0 \\
& \alpha_{12}-\alpha_{23}+\alpha_{13}<0
\end{aligned}
$$

Therefore,

$$
\alpha_{12}<0
$$

which 1s impossible. Hence, sin $A$, sin $B$, sin $C$ are all positive and consequently A, B, C are all positive. Thus,

$$
\begin{array}{ll}
\alpha_{12}+\alpha_{23} & >\alpha_{13} \\
\alpha_{12}+\alpha_{13} & >\alpha_{23} \\
\alpha_{23}+\alpha_{13} & >\alpha_{12}
\end{array}
$$

These relations show that $\alpha_{12}, \alpha_{23}$, and $\alpha_{13}$ satisfy the triangular inequality, and we have shown above that the sum of the three angles does not exceed $2 \pi$. Then there exist three points of the sphere of radius $r$ congruent to $p_{1}, p_{2}, p_{3}$

Theorem 11. Four points $\mathrm{p}_{2} \mathrm{p}_{2} \mathrm{p}_{3} \mathrm{p}_{4}$ of a semi-metric space are r-spheric if and only if each three of the points is $r$-spheric and $\Delta\left(p_{1}, p_{2}, p_{2}, p_{1}\right)=0$.

If the four points are r-spheric then each three is r-spheric and, by lomma 2, $\Delta\left(p_{1}, p_{2}, p_{3}, p_{4}\right)$ equals zero, and the necessity of the conditions is proved. 
To prove the eufficiency we suppose $\Delta\left(p_{1}, p_{2}, p_{3}, p_{4}\right)=0$ and that each three points is r-spheric, and we show there exist four points of the sphere congruent to $p_{1}, p_{2}, p_{3}, p_{4}$. The theorem is imediate if each three points is d-cyclic for then, by theorem 3, the four points are congruent to four points of a great circle of the sphere. We shall suppose, then, that at least one triple, say $p_{1}, p_{2}, p_{3}$, is not d-cyclic. Then no two of the pointe $p_{1}, p_{2}, p_{3}$ have the distance $T r_{3} i_{0} e_{0} \alpha_{02}$, $\alpha_{23}$, and $\alpha_{13}$ are all less than $\pi$. By hypothesis there exist three points, say $p_{1}^{\prime}, p_{2}^{\prime}, p_{3}^{\prime}$, of the sphere and three polnts, say $\bar{p}_{1}, \bar{p}_{2}, \bar{p}_{4}$, of the sphere such that

Then

$$
\begin{aligned}
& p_{1}, p_{2}, p_{3} \approx p_{1}^{\prime}, p_{2}^{\prime}, p_{3}^{\prime} \quad \text { and } \\
& p_{1}, p_{2}, p_{4} \approx \bar{p}_{1}, \bar{p}_{2}, \bar{p}_{4} .
\end{aligned}
$$

$\bar{p}_{1} \bar{p}_{2}=p_{1} p_{2}=p_{1} p_{2}$

Now we may make a congruent transformation of the sphere into itself so that $\bar{p}_{1}$ goes into $p_{1}^{\prime}$, and $\bar{p}_{2}$ goes into $p_{2}^{\prime} \cdot$. This transformation sends $\overline{p_{4}}$ into some point of the sphere, say $p_{4}^{*}$, such that $p_{1}^{\prime} p_{4}^{*}=p_{1} p_{4}$ and $p_{2}^{\prime} p_{4}^{*}=p_{2} p_{4} \cdot$. Since $p_{4}^{*}$ has its distances from the two points $p_{1}^{\prime}$ and $p_{2}^{\prime}$ determined and since these two points are not diametral, there are at most two such points $\mathrm{p}_{4}^{*}$. Tro cases present themselves.

Case 1. $p_{1} p_{2}, p_{1}$ are not d-cyclic. In this case there are exactly two possible positions on the sphere for $p_{4}^{*}$. We shall denote these points by $\mathrm{p}_{4}^{\Gamma}$ and $\mathrm{p}_{4}^{\Gamma}$. Then these two points are reflections of each other in the plane through $p_{1}^{\prime}$ and $p_{2}^{\prime}$ and the center of the sphere. Since $p_{1}, p_{2}, p_{3}$ are not d-cyclic, the point $p_{3}^{\prime}$ is not on this plane and hence $p_{3}^{\prime} p_{4}^{I} \neq p_{3}^{\prime} p_{4}^{\pi}$. We now have

$$
\begin{gathered}
p_{1}, p_{2}, p_{3} \approx p_{1}^{\prime}, p_{2}^{\prime}, p_{3}^{\prime} \\
p_{1}, p_{2}, p_{4} \approx p_{1}^{\prime}, p_{2}^{\prime}, \dot{p}_{4}^{I} \approx p_{1}^{\prime}, p_{2}^{\prime}, p_{4}^{\pi} .
\end{gathered}
$$


Hence, in order to prove the theorem we have only to show that $p_{3} p_{4}=$ $p_{3}^{\prime} p_{4}^{I}$ or $p_{3} p_{4}=p_{3}^{\prime} p_{4}^{I}$. In order to do this we define the expression $\triangle(x)$ as follows:

$$
\Delta(x)=\left|\begin{array}{cccc}
1 & \cos \alpha_{12} & \cos \alpha_{13} & \cos \alpha_{14} \\
\cos \alpha_{21} & 1 & \cos \alpha_{23} & \cos \alpha_{24} \\
\cos \alpha_{31} & \cos \alpha_{32} & 1 & \cos x \\
\cos \alpha_{41} & \cos \alpha_{42} & \cos x & 1
\end{array}\right|
$$

$\Delta(x)$ Is not identically zero for the coefflcient of $\cos ^{2} x$, namely - $\left(1-\cos ^{2} \alpha_{12}\right)$ does not vanish. By the necessity of the conditions of the theorem, $\Delta(x)=0$ has the two unequal roots $\frac{p_{3}^{\prime} p_{4}^{T}}{r}$ and $\frac{p_{3}^{\prime} p_{4}^{I}}{r}$. By hypothesis a root of $\Delta(x)=0$ is $\frac{p_{3 p 4}}{\sim}$. Since $\Delta(x)=0$ is a quadratic equation in $\cos x$, the equation has only two roots in the interval $0<x \leqslant \pi$. Hence either

$$
\begin{aligned}
& p_{3} p_{4}=p_{3}^{\prime} p_{4}^{I} \quad \text { or } \\
& p_{3} p_{4}=p_{3}^{\prime} p_{4}^{I} .
\end{aligned}
$$

In either case the theorem is proved, for either

$$
\begin{aligned}
& p_{1}, p_{2}, p_{3}, p_{4} \approx p_{1}^{\prime}, p_{2}^{\prime}, p_{3}^{\prime}, p_{4}^{ \pm} \text {or } \\
& p_{1}, p_{2}, p_{3}, p_{4} \approx p_{1}^{\prime}, p_{2}^{\prime}, p_{3}^{\prime}, p_{4}^{\prime \prime} \text {. }
\end{aligned}
$$

Case 2. $\mathrm{p}_{1}, \mathrm{p}_{2}, \mathrm{p}_{4}$ are d-eyclic. In this case the point $\mathrm{p}_{4}$ is unique. We shall denote this point by $p_{4}^{\prime}$ and shall show that

$$
p_{1}, p_{2}, p_{3}, p_{4} \approx p_{1}^{\prime} p_{2}^{\prime} p_{3}^{\prime}, p_{4}^{\prime}
$$

We have

$$
\begin{aligned}
& p_{1}, p_{2}, p_{3} \approx p_{1}^{\prime}, p_{2}^{\prime}, p_{3}^{\prime} \quad \text { and } \\
& p_{1}, p_{2}, p_{4} \approx p_{1}^{\prime}, p_{2}^{\prime}, p_{4}^{\prime} \text {. }
\end{aligned}
$$

Thus we need only to show that $p_{3} p_{4}=p_{3}^{1} p_{4}^{1}$ - To do this we define the expression $\Delta(x)$ as in the preceding case and we show that $\frac{p_{3} p_{4}}{r}$ 


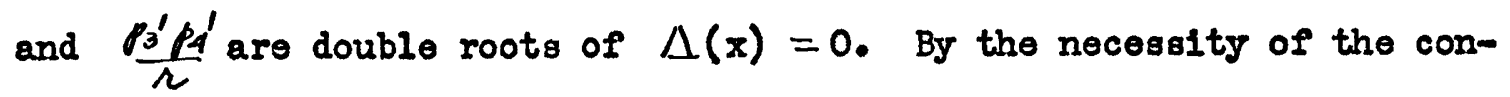
ditions of the theorem a root of $\Delta(x)=0$ is $\frac{p_{3}^{\prime} P_{4}^{\prime}}{\Lambda}$. By hypothesis a root of $\Delta(x)=0$ is $\frac{p_{3} p_{4}}{n}$. Expanding $\Delta(x)$ wo obtain

$$
\Delta(x)=\frac{-\left|\begin{array}{ccc}
1 & \cos \alpha_{12} & \cos \alpha_{13} \\
\cos \alpha_{21} & 1 & \cos \alpha_{23} \\
\cos \alpha_{41} & \cos \alpha_{42} & \cos x
\end{array}\right|^{2}}{1-\cos ^{2} \alpha_{12}}
$$

$\Delta(x)$ is not identically zero for the same reason as in the preceding. case, and $\Delta(x)=0$ has only two roots in the interval $0<x \leq \pi$. From

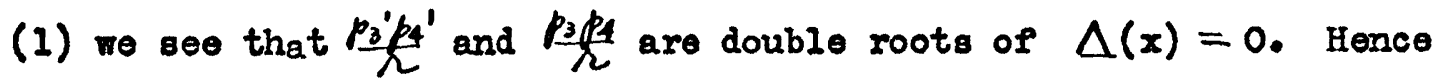
$p_{3} p_{4}=p_{3}^{\prime} p_{4}^{\prime} \cdot$ Therefore

$$
p_{1}, p_{2}, p_{3}, p_{4} \approx p_{1}^{\prime}, p_{2}^{\prime}, p_{3}^{\prime}, p_{4}^{\prime}
$$

and the proof is completed.

Theorem 12. Five points $D_{2}, D_{2}, P_{3}, P_{4}, D_{5}$ of a semi-metric space are r-spheric if and only if each four of the points is $r$-spheric and $\Delta\left(p_{1}, p_{2} p_{3}, p_{4}, p_{3}\right)=0$

If the five points are r-spheric then obviously each four of the points is i-ephèric. By lemma 2, $\Delta\left(p_{1}, p_{2}, p_{3}, p_{4}, p_{5}\right)=0$. Hence, the necessity of the conditions is established.

By theorem 11 each fourth-order principal minor vanishes. If each three points is d-cyclic, then by theorem 3 each four points is dcyclic. The suffictency of the conditions is immediate in this case since the circle has the congruence order four. In this case the five points are congruent to five points of a great circle of the sphere. Now we need only to establish the sufficiency of the conditions for the case in which at least one triple is r-spheric and not d-cyclic. We may assume the labeling so that $p, p_{2}, p_{3}$ are not d-cyclic. Then $\Delta\left(p_{1}, p_{2}, p_{3}\right)$ is positive. By hypothesis there exist four points, say 
$p_{1}^{\prime}, p_{2}^{\prime}, p_{3}^{\prime}, p_{4}^{\prime}$ of the aphere and four points, say $\bar{p}_{1}, \bar{p}_{2}, \bar{p}_{3}, \bar{p}_{5}$, of the sphere such that

$$
\begin{aligned}
& p_{1}, p_{2}, p_{3}, p_{4} \approx p_{1}^{\prime}, p_{2}^{\prime}, p_{3}^{\prime}, p_{4}^{\prime} \text { and } \\
& p_{1}, p_{2}, p_{3}, p_{5} \approx \bar{p}_{1}, \bar{p}_{2}, \bar{p}_{3}, \bar{p}_{5} .
\end{aligned}
$$

To may make a congruent transformation of the sphere into itself so that $\bar{p}_{1}$ goes into $p_{1}^{\prime}, \bar{p}_{2}$ goes into $p_{2}^{\prime}, \bar{p}_{3}$ goes into $p_{3}^{\prime}$, and $\bar{p}_{5}$ goes into some point, say $p_{5}^{\prime}$. The point $p_{5}^{\prime}$ has its distances from the three pointe $p_{1}^{\prime}, p_{2}^{\prime}, p_{3}^{\prime}$ fixed and equal respectively to $p_{1} p_{3} p_{2} p_{5}$ and $p_{3} p_{5}$. Nor $p_{1}^{\prime}, p_{2}^{\prime}, p_{3}^{\prime}$ are not on a great circle of the ephere because $p_{1}^{\prime}, p_{2}^{\prime}, p_{3}^{\prime}$ are congruent to $p_{1}, p_{2}, p_{3}$ and this triple is not d-cyclic. Hence the point $p_{5}^{\prime}$ is unique. We wish to show that

$$
p_{1}, p_{2}, p_{3}, p_{4}, p_{5} \approx p_{1}^{\prime}, p_{2}^{\prime}, p_{3}^{\prime}, p_{4}^{\prime}, p_{5}^{\prime} \text {. }
$$

We have

$$
\begin{aligned}
& p_{1}, p_{2}, p_{3}, p_{4} \approx p_{1}^{\prime}, p_{2}^{\prime}, p_{3}^{\prime}, p_{4}^{1} \quad \text { and } \\
& p_{1}, p_{2}, p_{3}, p_{5} \approx p_{1}^{\prime}, p_{2}^{\prime}, p_{3}^{\prime}, p_{5}^{\prime} \text {. }
\end{aligned}
$$

Hence we need only to show that $p_{4} p_{5}=p_{4}^{\prime} p_{5}^{\prime}$.

In order to do this we define the expression $\Delta(x)$ as follows:

$$
\Delta(x)=\left|\begin{array}{ccccc}
1 & \cos \alpha_{12} & \cos \alpha_{13} & \cos \alpha_{14} & \cos \alpha_{15} \\
\cos \alpha_{21} & 1 & \cos \alpha_{23} & \cos \alpha_{24} & \cos \alpha_{25} \\
\cos \alpha_{31} & \cos \alpha_{32} & 1 & \cos \alpha_{34} & \cos \alpha_{35} \\
\cos \alpha_{41} & \cos \alpha_{42} & \cos \alpha_{43} & .1 & \cos x \\
\cos \alpha_{51} & \cos \alpha_{52} & \cos \alpha_{53} & \cos x & 1
\end{array}\right|
$$

$\Delta(x)$ does not vanish identically, since the coefficient of $\cos ^{2} x$, namely $-\Delta\left(p_{1}, p_{2}, p_{3}\right)$ is not zero. By the necessity of the conditions of the theorem a root of $\Delta(x)=0$ is $p_{1}^{\prime} p_{5}^{\prime}$. By hypothesis a root of $\Delta(x)=0$ is $\frac{p+p}{2}$. Since $\Delta(x)=0$ is a quadratic equation in $\cos x$ the equation has only two roots in the interval $0<x \leq \pi$. Expanding $\Delta(x)$ we have 


$$
\Delta(x)=\frac{-\left|\begin{array}{cccc}
1 & \cos \alpha_{12} & \cos \alpha_{13} & \cos \alpha_{14} \\
\cos \alpha_{21} & 1 & \cos \alpha_{23} & \cos \alpha_{24} \\
\cos \alpha_{31} & \cos \alpha_{32} & 1 & \cos \alpha_{34} \\
\cos \alpha_{51} & \cos \alpha_{52} & \cos \alpha_{53} & \cos x
\end{array}\right|^{2}}{\Delta\left(p_{1}, p_{2}, p_{3}\right)}
$$

Hence $\frac{p_{t}^{\prime} p s^{\prime}}{r}$ and $\frac{p_{1} p_{5}}{r}$ are double roots of $\Delta(x)=0$. Therefore

$$
\begin{gathered}
p_{4} p_{5}=p_{4}^{\prime} p_{5}^{\prime} \text { and hence } \\
p_{1}, p_{2}, p_{3}, p_{4}, p_{5} \approx p_{1}^{\prime}, p_{2}^{\prime}, p_{3}^{\prime}, p_{4}^{\prime}, p_{5}^{\prime}
\end{gathered}
$$

which is what we wished to show.

Theorems 10, 11, and 12 characterize r-spheric triples, quadruples, and quintuples. Since the sphere has the congruence order five*, it follows that the necessary and sufficient condition that a set of points be $r$-spheric is that each quintuple of the set satisfy the conditions stated in theorem 12: The remainder of this section consists of a treatment pseudo r-spheric sets containing fire points. Theorem 13. If five points $p_{1}, p_{2}, p_{3}, p_{4}, p_{8}$ of a semi-metric space are rsoudo $r$-spheric then $\Delta\left(p, 2 p, 2, p_{2}, p_{,}\right)$is netative. From the definition of the pseudo r-spheric quintuple and thoorem 11 each fourth-order principal minor of $\Delta\left(p_{1}, p_{2}, p_{3}, p_{4}, p_{5}\right)$ vanishes. Hence, at least one third-order principal minor does not raniah; that is, at least one triple is not d-cyclic, for otherwise each four points is d-cyclic by theorem 3. But if each four points is d-cyclic, the fire points are d-cyclic, since the circle has the congruence order four, and hence are r-spheric. Thus, we may

* Menger, Loc. cit., p. 725.

IIt is obvious that if $p_{1}, p_{2}, \ldots, p_{n}(n>5)$ are mopheric, then $\Delta\left(p_{1}, p_{2}, \ldots, p_{n}\right)=0$. Ce. Bocher, Loc. cit. 
assume the labeling so. that $p_{1}, p_{2}, p_{3}$ are r-spheric and are not d-cyclic. Hence

$$
\Delta\left(p_{1}, p_{2}, p_{3}\right)>0
$$

Evaluating $\Delta\left(p_{1}, p_{2}, p_{3}, p_{4}, p_{5}\right)$ we obtain

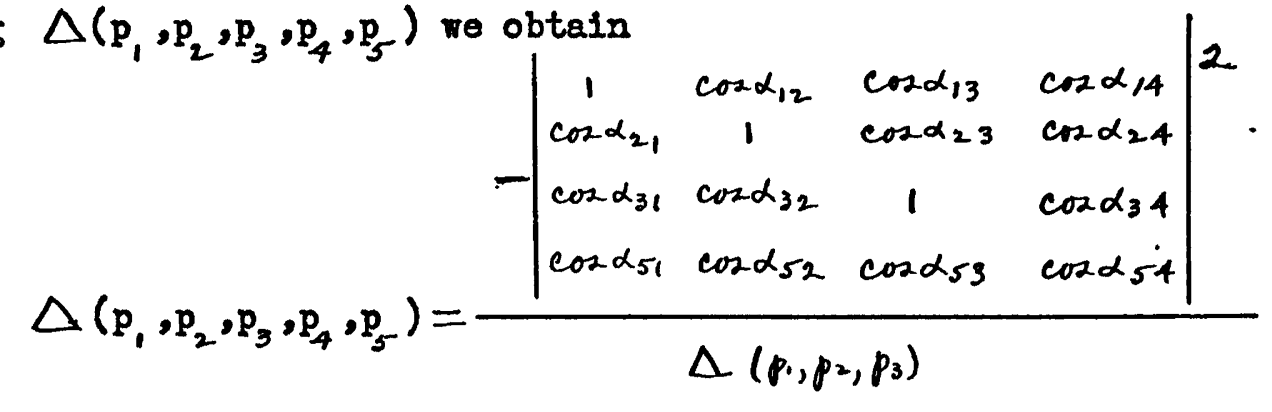

Therefore $\Delta\left(p_{1}, p_{2}, p_{3}, p_{4}, p_{5}\right) \leq 0$.

But if $\Delta\left(p_{1}, p_{2}, p_{3}, p_{4}, p_{5}\right)$ equals zero, the five points are r-apheric by the preceding theorem. Hence

$$
\Delta\left(p_{1}, p_{2}, p_{3}, p_{4}, p_{5}\right)<0
$$

Theorem 14. None of the triples contained in a poeudo $r$-apheric guintuple is d-cyclic and each two points are isogonal onconjugates With respect to the other three points.

We shall let the pseudo r-apheric quintuple be $p_{1}, p_{2}, p_{3}, p_{4}, p_{5}$. As was shown in the proof of theorem 13, at least one triple contained in these points is not d-cyclic. We may assume the labeling so that $p_{1}, p_{2}, p_{3}$ is such a triple. There exist three points $p_{1}^{\prime}, p_{2}^{\prime}, p_{3}^{\prime}$ of the sphere such that

$$
p_{1}, p_{2}, p_{3} \approx p_{1}^{\prime}, p_{2}^{\prime}, p_{3}^{\prime}
$$

Then $p_{1}^{\prime}, p_{2}^{\prime}, p_{3}^{\prime}$ are not on a freat circle. There exist four points $p_{1}^{\prime \prime}, p_{2}^{\prime \prime}, p_{3}^{\prime \prime}, p_{4}^{\prime \prime}$ of the sphere such that

$$
p_{1}, p_{2}, p_{3}, p_{4} \approx p_{1}^{\prime \prime}, p_{2}^{\prime \prime}, p_{3}^{\prime \prime}, p_{4}^{\prime \prime}
$$

Hence

$$
p_{1}, p_{2}, p_{3} \approx p_{1}^{\prime \prime}, p_{2}^{\prime \prime}, p_{3}^{\prime \prime} \approx p_{1}^{\prime}, p_{2}^{\prime}, p_{3}^{\prime} \text {. }
$$

Tie may make a congruent transformation of the sphere into itself 80 that $p_{1}^{\prime \prime}$ goes into $p_{1}^{\prime}, p_{2}^{\prime \prime}$ goe's into $p_{2}^{\prime}, p_{3}^{\prime \prime}$ goes into $p_{3}^{\prime}$, and $p_{4}^{\prime \prime}$ 
goes into some point, say $p_{4}^{\prime}$ - Similarly, there exist four points, say $p_{1}^{\prime \prime \prime}, p_{2}^{\prime \prime \prime}, p_{3}^{\prime \prime \prime}, p_{5}^{\prime \prime \prime \prime}$ of the sphere such that

$$
p_{1}, p_{2}, p_{3}, p_{5} \approx p_{1}^{\prime \prime \prime}, p_{2}^{\prime \prime \prime}, p_{3}^{\prime \prime \prime}, p_{5}^{\prime \prime \prime} \text {. }
$$

He may make a congruent transformation of the sphere into itself so that $p_{1}^{\prime \prime \prime}$ goes into $p_{1}^{\prime}, p_{2}^{\prime \prime \prime}$ goes into $p_{2}^{\prime}, p_{3}^{\prime \prime \prime}$ goes into $p_{3}^{\prime}$, and $p_{5}^{\prime \prime \prime}$ goes into some point, say $p_{5}^{\prime}$. The points $p_{4}^{\prime}$ and $p_{5}^{\prime}$ so determined are unique since their distances from the three foints $p_{1}^{\prime}, p_{2}^{\prime}, p_{3}^{\prime}$ are fixed. and since these three points are not on a great circle of the sphere. Thus we have

$$
\begin{aligned}
& p_{1}, p_{2}, p_{3}, p_{4} \approx p_{1}^{\prime}, p_{2}^{\prime}, p_{3}^{\prime}, p_{4}^{\prime} \\
& p_{1}, p_{2}, p_{3}, p_{5} \approx p_{1}^{\prime}, p_{2}^{\prime}, p_{3}^{\prime}, p_{5}^{\prime}
\end{aligned}
$$

Hence

$$
\mathrm{p}_{4} \mathrm{p}_{5} \neq \mathrm{p}_{4}^{\prime} \mathrm{p}_{5}^{\prime}
$$

for otherwise $p_{1}, p_{2}, p_{3}, p_{4}, p_{5}$ are congruent to $p_{1}^{\prime}, p_{2}^{1}, p_{3}^{1}, p_{4}^{\prime}, p_{5}^{\prime}$ and thus are r-spheric, which is impossible.

There exist four points, sayt $\bar{p}_{2}, \bar{p}_{3}, \bar{p}_{4}, \bar{p}_{5}$, of the sphere such that

$$
\mathrm{p}_{2}, \mathrm{P}_{3}, \mathrm{p}_{4}, \mathrm{p}_{5} \approx \overline{\mathrm{p}}_{2}, \overline{\mathrm{p}}_{3}, \overline{\mathrm{p}}_{4}, \overrightarrow{\mathrm{P}}_{5}
$$

Then

$$
\mathrm{p}_{2}, \mathrm{p}_{3}, \mathrm{p}_{5} \approx \overline{\mathrm{p}}_{2}, \overline{\mathrm{p}}_{3}, \overline{\mathrm{p}}_{5} \approx \mathrm{p}_{2}^{1}, \mathrm{p}_{3}^{\prime}, \mathrm{p}_{5}^{\prime} \text {. }
$$

We may make a congruent transfornation of the sphere into itself sending $\bar{p}_{2}$ into $p_{2}^{1}, \bar{p}_{3}$ into $p_{3}^{1}$, and $\bar{p}_{5}$ into some point, say $p_{5}^{1} \cdot$ Hence we have

$$
\mathrm{p}_{2}, \mathrm{p}_{3}, \mathrm{p}_{4}, \mathrm{p}_{5} \approx \mathrm{p}_{2}^{\prime}, \mathrm{p}_{3}^{\prime}, \mathrm{p}_{4}^{\prime}, \mathrm{p}_{5}^{1}
$$

From (1), (2), (3), and (4)

$$
\begin{aligned}
& p_{2}^{\prime} p_{5}^{1}=p_{2} p_{5}=p_{2}^{\prime} p_{5}^{\prime} \\
& p_{3}^{\prime} p_{5}^{1}=p_{3} p_{5}=p_{3}^{\prime} p_{5}^{\prime} \\
& p_{4}^{\prime} p_{5}^{1}=p_{4} p_{5} \neq p_{4}^{1} p_{5}^{\prime} \cdot
\end{aligned}
$$

Therefore $p_{5}^{1}$ and $p_{5}^{1}$ are distinct and $p_{5}^{1}$ is the image of $p_{5}^{\prime}$ in the plane through $p_{2}^{\prime}, p_{3}^{\prime}$, and the center of the sphere. Treating in a similar 
manner the two remaining quadruples; namely, $p_{1}, p_{3}, p_{4}, p_{5}$ and $p_{1}, p_{2}, p_{4}, p_{5}$ we obtain the relations

$$
\begin{aligned}
& p_{1}, p_{3}, p_{4}, p_{5} \approx p_{1}^{\prime}, p_{3}^{\prime}, p_{4}^{\prime}, p_{5}^{2} \\
& p_{1}, p_{2}, p_{4}, p_{5} \approx p_{1}^{\prime}, p_{2}^{\prime}, p_{4}^{\prime}, p_{5}^{3}
\end{aligned}
$$

where $p_{5}^{2}$ is the image of $p_{5}^{1}$ in the plane through $p_{1}^{1}, p_{3}^{\prime}$, and the center of the sphere, and $p_{5}^{3}$ is the image of $p_{5}^{\prime}$ in the plane through $p_{1}^{\prime}, p_{2}^{\prime}$, and the center of the sphere.

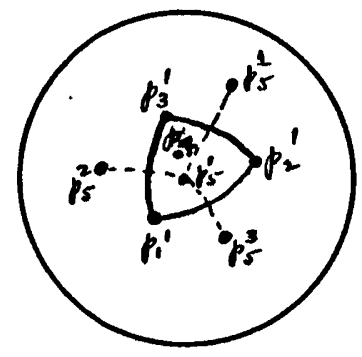

Fig. 2

From (2), (3), (4), and (5) we have

$$
p_{4} p_{5}=p_{4}^{\prime} p_{5}^{1}=p_{4}^{\prime} p_{5}^{2}=p_{4}^{\prime} p_{5}^{3} \neq p_{4}^{\prime} p_{5}^{\prime} .
$$

Thus $p_{4}^{\prime}$ is equidistent from the points $p_{5}^{1}, p_{5}^{2}$, and $p_{5}^{3}$, which are, respectively, the images of $p_{5}^{\prime}$ in the plane through $p_{2}^{\prime}, p_{3}^{\prime}$, and the center of the sphere; $p_{1}^{\prime}, p_{3}^{\prime}$, and the center of the sphere; and $p_{1}^{\prime}$, $p_{2}^{\prime}$, and the center of the sphere. Therefore $p_{4}^{1}$ is the isogonal conjugate of $p_{5}^{1}$ with respect to $p_{1}^{1}, p_{2}^{1}, p_{3}^{1}$.

The above analyais 1 tself may be used to show that $p_{1}, p_{2}, p_{5}$ are not d-cyclic, for if 80 , then $p_{1}^{\prime}, p_{2}^{\prime}, p_{s}^{\prime}$ are on a great circle and the points $p_{4}^{\prime}$ and $p_{3}^{\prime}$ must be identical, which is impossible. Likewise $p_{1}, p_{3}, p_{5}$ and $p_{2}, p_{3}, p_{5}$ are not d-cyclic. Now we may use $p_{1}, p_{2}, p_{5}$ in place of $p_{1}, p_{2}, p_{3}$ in the analysis above. In this manner we find $p_{4}^{\prime}$ and $p_{3}^{\prime}$ are isogonal conjugates with respect to $p_{1}^{\prime}, p_{2}^{\prime}, p_{5}^{\prime}$. Using $p_{1}, p_{3}, p_{5}$ in place of $p_{1}, p_{2}, p_{3}$ we find that $p_{4}^{\prime}$ and $p_{2}^{\prime}$ are isogonal conjugates with respect to $p_{1}^{\prime}, p_{3}^{\prime}, p_{5}^{\prime}$ and so on. Finally, we find that each two 
points are isogonal conjugates with respect to the other three points and that no three points are d-cyclic, for if three of the points are d-cyclic then the isogonal conjugate construction breaks down, two of the points being identical, which is impossible. 